\title{
Factores relevantes del desarrollo profesional y de compensaciones en la carrera laboral del trabajador ${ }^{*}$
}

Sergio Manuel

Madero Gómez.

Escuela de Negocios, ITESM, campus Monterrey

smadero@itesm.mx
*El autor agradece la valiosa participación y aportaciones en este trabajo de Abel Anaya, Esthela Rivera, Irma Peña, Karla Ruiz y Verónica Herrera, participantes del Programa de Posgrado del Cetys Universidad, Campus, Mexicali

\section{Resumen}

En la actualidad el desarrollo profesional y las compensaciones son temas relevantes dentro de la administración del personal en las empresas; por ello, en esta investigación se ha analizado un conjunto de 27 factores, que están relacionados con las áreas de recursos humanos, para poder conocer la importancia que tienen en la carrera laboral de las personas. Se recolectaron 348 cuestionarios mediante una encuesta electrónica, utilizando el software NCSS para su análisis. Entre los principales resultados destaca que los factores más sobresalientes en la carrera laboral para las mujeres mexicanas son el nivel educativo, las habilidades de comunicación y el apoyo familiar; mientras que para las mujeres estadounidenses son las habilidades para equilibrar su vida profesional y familiar, habilidades interpersonales y habilidades de comunicación.

Palabras claves: desarrollo profesional, desarrollo de carrera, compensaciones, recursos humanos, género. 


\section{Relevant factors of professional development and compensations for worker's career}

At present professional development and compensation are relevant issues for the administration of personnel areas in business. This investigation analyzes a set of 27 factors associated with these areas of human resources in order to know how relevant those factors are for the careers of individuals. 348 questionnaires were collected through an electronic survey, using the NCSS software for the analysis. Among the mayor results found it stands out that the most relevant factors in the career for Mexican women are: education, communication skills and family support, while for U.S. women they are: ability to balance their professional and family life, interpersonal skills and communication skills.

Keywords: professional development, career development, compensation, human resources, gender.

\section{Introducción}

El enfoque principal de este estudio es determinar las diferencias de los factores relacionados con el desarrollo profesional y las compensaciones entre los hombres y las mujeres que trabajan de la zona fronteriza de Mexicali, Baja California. Para llevar a cabo este trabajo se tomaron como base los estudios realizados por Marlow, Marlow y Arnold (1995) y Syed Tahir Hijazi, Adeel Anwar y Syed Ali Abdullah Mehboob (2007), pues contribuyen a profundizar en el estudio de estos temas relevantes en el manejo de personal en las empresas.

El interés por realizar este trabajo es conocer cuáles son los factores más importantes relacionados con el desarrollo profesional y con las compensaciones que se otorgan a las personas durante su trayectoria laboral en el entorno mexicano. Esto con la finalidad de mostrar los resultados a directivos y gerentes de las empresas para que contribuyan en el diseño, desarrollo e implementación de estrategias y proyectos de desarrollo profesional, así como en mejores prácticas de compensaciones y programas enfocados a mejorar la motivación y satisfacción laboral. Con lo anterior se logrará establecer esquemas de mejora en las actividades propias del área de recursos humanos dentro del medio empresarial. 


\section{Marco teórico}

El desarrollo de la carrera es el interés que demuestra la compañía en el crecimiento de los colaboradores debido a que tiene una influencia directa sobre éste mediante diversas herramientas como planes y rutas de carrera, entre otras, para fortalecer los lazos de unión y la retención de los empleados (Chiavenato, 2007: 410-412; Gómez Mejía, Balkin y Cardy, 2001: 320.)

Es así que un factor importante por considerar en el diseño y desarrollo de la carrera de los empleados es el impacto que tiene la satisfacción, el compromiso y el desempeño de la persona en el puesto, tal como lo indican McCloskey e Igbaria (2003: 30-31), Igbaria y Shano (1997: 16-17), Igbaria y Greenhaus (1992: 488-493). De igual forma, otro factor por considerar es que la mayoría de los profesionales que actualmente están trabajando son jóvenes menores de 30 años, quienes se encuentran en la búsqueda de perspectivas de carrera dentro de las empresas más que de un pago más atractivo, pues su principal interés es la posibilidad de encontrar una serie de oportunidades de crecimiento y desarrollo.

Indudablemente, si las personas encuentran dentro de la empresa posibilidades de promoción en su carrera y crecimiento profesional se sentirán más satisfechas y permanecerán colaborando más tiempo, lo que originará que contribuyan indudablemente con los resultados de la organización (Madero, 2009). La influencia de la organización sobre el compromiso de conservación de los empleados y la productividad está en concordancia con estudios anteriores (Mowday et al., 1982). Silverman (2006) menciona las áreas o actividades más relevantes que deben considerarse para incrementar el desarrollo profesional en los empleados. A continuación se detalla cada una de ellas.

1) Construir relaciones fuertes entre los mismos empleados y entre el binomio jefe-colaborador debido a que cuando los empleados perciben que tienen a su mejor amigo en el trabajo se puede decir que son más productivos, más positivos con los clientes, comparten nuevas ideas y permanecen más tiempo en su trabajo.

2) Desarrollar el potencial individual. Consiste en implementar un proceso de comunicación entre el personal; para ello, se efectúan preguntas personalizadas acerca de lo que es importante para su crecimiento y desarrollo en el trabajo. 
3) Promover la colaboración y el trabajo en equipo. Con la finalidad de poder generar mayor integración entre los diversos integrantes del equipo y así poder unir sus esfuerzos en la búsqueda de sus objetivos en común.

4) Conectar a todos hacia la misma dirección. Mientras las relaciones personales se estrechen y vinculen se espera que se desarrolle el potencial y se promueva la colaboración y el trabajo en equipo, ayudando a construir un mayor compromiso; sin embargo, las personas necesitan conocer la visión y las metas de la organización.

A continuación se mencionan los diversos conceptos que Syed et al. (2007) contemplan y que se tomaron en consideración para el diseño y desarrollo de nuestra investigación.

Las recompensas se clasifican dentro de dos categorías: recompensas financieras que son aquellas otorgadas en términos monetarios y tienen valor monetario, y las recompensas no financieras que provienen del propio trabajo, del ambiente laboral y que no tienen ningún valor monetario (Bohlander, Snell y Sherman, 2001: 356; Menefee y Murphy, 2004: 17; Schuster y Zingheim, 1993: 27; Zobal, 1998: 240 y 1999: 27-32; Gómez Mejía, Balkin y Cardin, 2001: 352; y Mondy y Noe, 2005: 285).

Recompensas financieras. También conocidas como compensaciones monetarias, normalmente incluyen el sueldo base, pagos por desempeño o contribución, pagos variables (bonos) y otros beneficios e incentivos financieros. Estas recompensas son clasificadas como recompensas transaccionales porque se otorgan con base en los resultados entre el empleado y el empleador.

Recompensas no financieras. Se conocen como compensaciones no monetarias, normalmente incluyen el reconocimiento, la responsabilidad, el trabajo significativo, la autonomía, la oportunidad para desarrollar las habilidades, oportunidades de carrera, calidad de vida y equilibrio de trabajo y vida. Estas recompensas se clasifican como recompensas relacionales porque están relacionadas con aprendizaje, desarrollo y la experiencia laboral de los trabajadores. Normalmente, se considera que las recompensas no financieras son otorgadas para complementar las recompensas financieras, pero éstas también tienen importancia y significado en la motivación y productividad de los trabajadores. 
Generalmente, las organizaciones recompensan a sus empleados para incrementar su nivel de motivación y para acrecentar su desarrollo y productividad. Las recompensas y compensaciones organizacionales son las que el empleado obtiene en pago a sus esfuerzos y al trabajo que realiza para contribuir al logro de las metas organizacionales. Caligiuri y Cascio (2000) y Cascio (2003) mencionan que la compensación incluye pagos directos en efectivo, pagos indirectos en forma de beneficios para el empleado e incentivos para motivar a los empleados a esforzarse para obtener niveles altos de productividad.

Por lo que respecta a los factores importantes para el desarrollo de carrera, tomamos en cuenta los que Marlow, Marlow y Arnold (1995) mencionan en su trabajo; podemos destacar que uno de los factores que facilita el desarrollo profesional es el "tener un mentor" (Roche, 1979; Kaye y Robinson, 1997; Kaye y Jacobson, 1996; Dalton, Thompson y Price, 1977).

De igual forma, es importante mencionar que existe una serie de habilidades o competencias que las personas deben tener o desplegar para que su desarrollo profesional al momento de realizar sus actividades sea lo más destacado posible; por ejemplo, que las habilidades de comunicación le permitan relacionarse con los demás y que posean las habilidades para adaptarse a las diversas situaciones que surgen diariamente.

Por otra parte, en la actualidad lo que inhibe el desarrollo profesional de la mujer no es la falta de habilidades, sino el hecho de que un supervisor hombre no puede lidiar con un empleado que no cumple con el modelo tradicional masculino (Domínguez, 1992; Greenhaus, Parasuraman y Wormley, 1990). Aunado a ello, otro factor que obstaculiza el desarrollo profesional del sexo femenino son las obligaciones familiares (Andrew, Coderre y Denis, 1990; Kelly y Guy, 1991; Wentling, 1992; Wentling, 1993). A diferencia de los hombres, se espera que la mujer desempeñe dos papeles: uno en la oficina y otro en la casa.

Los resultados de un estudio indicaron que las mujeres que tienen obligaciones familiares menores son las que se desempeñan mejor en niveles altos de supervisión (Kelly y Guy, 1991). Se afirma que ellas sacrifican su vida personal para ser exitosas en el trabajo o sacrifican su carrera por sus familias. Brennan (1992) encontró que las mujeres están mucho más dispuestas a cambiar de residencia para ayudar en la carrera de sus parejas que los hombres. El problema con las obligaciones familiares es exacerbado por la suposición de que las mujeres con hijos están menos comprometidas con su trabajo (Hamilton, 1993; Naff, 1994). 
Otro factor que obstaculiza el desarrollo profesional en las empresas consiste en que se carece de planes de carrera formalmente establecidos para los trabajadores, tal como lo mencionan Margol (1993) y Hamilton (1993). También hay que mencionar que en la actualidad, el problema no es que las mujeres no planeen sus actividades enfocadas para su desarrollo profesional, sino que sus carreras no cubren el modelo tradicional que se tiene desde la perspectiva masculina en las empresas (Jaffe, 1985).

\section{Planteamiento del problema}

En el área laboral se han investigado los factores que afectan al individuo en su desarrollo dentro de una organización con el objeto de identificar cuáles son los que más repercuten en el resultado que las empresas buscan; es decir, una mayor productividad se traduce en mayores ganancias. Con la identificación del nivel de importancia de dichos factores, los departamentos de recursos humanos y de desarrollo pueden hacer uso de éstos para crear nuevos planes de carrera del personal que está iniciando vida laboral; crear atractivos planes de incentivos monetarios, mejorar sus jornadas de trabajo y, por ende, su calidad de vida.

Por esta razón, se investigó cuáles son los principales factores relacionados con el desarrollo de carrera y con las compensaciones de tipo monetario y no monetario que más influyen en los profesionistas mexicanos para comprobar si hay diferencias en cuanto al género de los participantes.

\section{Objetivos de la investigación}

Los objetivos de esta investigación son los siguientes:

- Conocer la importancia de los diferentes criterios del desarrollo profesional de acuerdo con el género de las personas.

- Conocer las diferencias de acuerdo con el género de los diversos criterios relacionados con el desarrollo profesional.

- Conocer las diferencias de acuerdo con el género de los diversos criterios relacionados con las compensaciones monetarias y no monetarias.

- Conocer si existe alguna relación entre los factores de desarrollo profesional y los factores monetarios y no monetarios. 


\section{Modelo particular de investigación}

De acuerdo con los planteamientos anteriores se diseñó el modelo particular para la investigación; para ello se consideraron los 18 factores que Marlow, Marlow y Arnold (1995) desarrollan en su trabajo; además, considerando la relevancia que tienen los esquemas de pagos en los diversos procesos de recursos humanos, hemos incluido nueve factores más relacionados con la gestión de compensaciones (véase figura 1).

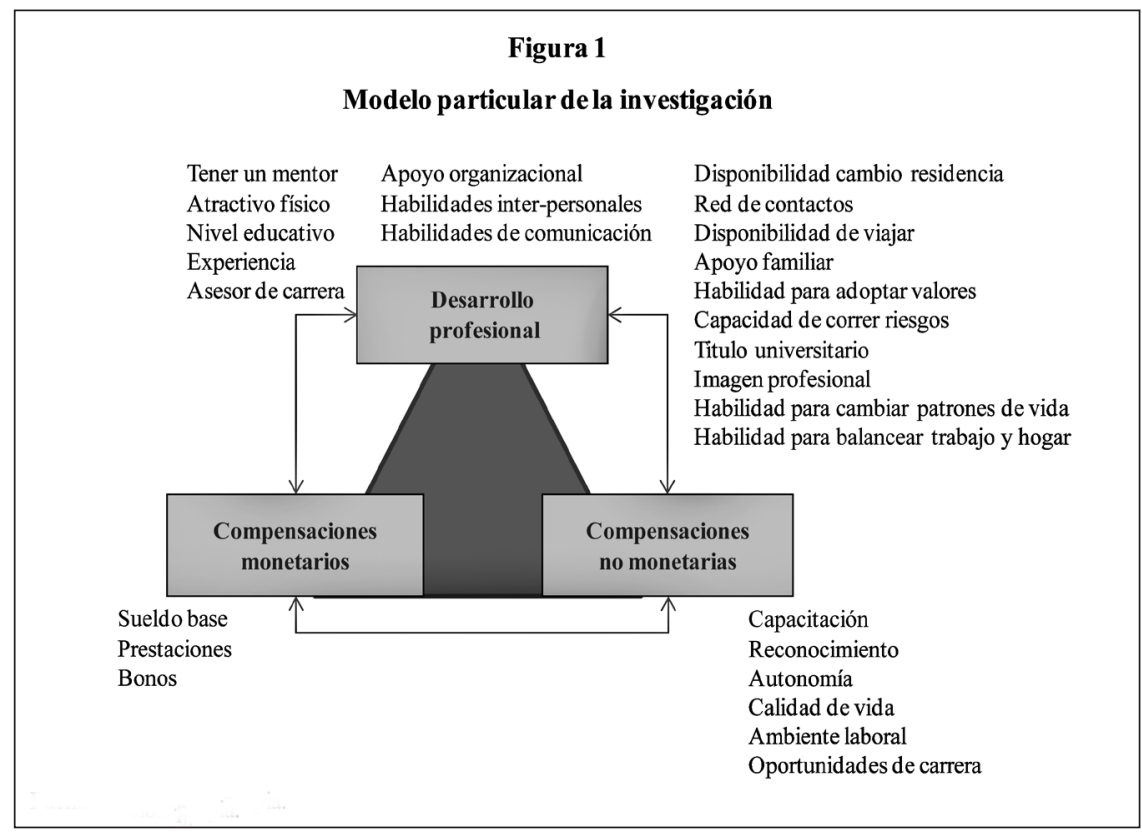

\section{Metodología}

Se tomó en cuenta el trabajo realizado por Marlow, Marlow y Arnold (1995) en el que utilizaron una muestra aleatoria de mil mujeres de alto nivel jerárquico que trabajan en empresas de Estados Unidos; el cuestionario se envió por correo postal, obteniendo una respuesta de 170 cuestionarios, en el que incluía 18 variables relacionadas con el desarrollo profesional en escala Likert de 1 a 5, donde 5 indica que el factor es extremadamente importante. 
Para el desarrollo de la presente investigación de tipo no experimental transaccional, correlacional, el método para la selección de la muestra fue no probabilístico, utilizando un muestreo por conveniencia (Hernández-Sampieri, Fernández y Baptista, 2006). La unidad de estudio fueron personas que estuvieran trabajando; no se consideró género, edad, área de trabajo, nivel jerárquico, pertenecientes al sector público o privado en la ciudad de Mexicali, Baja California. La recolección de datos se realizó mediante aplicación de cuestionario autoadministrado por correo electrónico.

El instrumento de medición contenía 34 enunciados, de los cuales 27 corresponden a variables que se presentan en el modelo particular y se valoraron con base en escala Likert de 1 a 5 para indicar el grado de importancia que las personas otorgan a cada una de ellas, donde 1 es el de menor grado y 5 el de extrema importancia; los siete enunciados restantes corresponden a los datos demográficos de las personas participantes.

\section{Estrategia de recolección de datos}

Se enviaron mil correos electrónicos con un anexo que contenía un documento donde se explicaba el objetivo del estudio; además, se solicitó a los participantes que accesaran a una página Web en la que se localizaba el cuestionario. Dado que en los primeros tres días no se observó una respuesta favorable, se decidió enviarles un recordatorio; se les dio una semana de tiempo adicional para contestar. Se obtuvieron 348 cuestionarios que representaba un $34.8 \%$ del total. Es importante señalar que los resultados obtenidos en esta investigación corresponden únicamente a la muestra señalada, por lo que no pueden ser generalizados a toda la población. El análisis estadístico de los datos se efectuó mediante los programas SPSS y NCSS 2007.

\section{Análisis de los resultados}

\section{Características de la muestra}

De las 348 personas que contestaron el cuestionario, 167 son hombres y $181 \mathrm{mu}-$ jeres; según la experiencia laboral, el 57\% tiene menos de 5 años y el 23\% entre 6 y 10 años. Con respecto al nivel de escolaridad, predominan las personas con carrera profesional (57\%) y estudios de posgrado (17\%). En cuadro 1 de la sección de anexos, se puede observar la información con los datos demográficos de la muestra. 
Comparación de mujeres y hombres de acuerdo con los criterios para el desarrollo profesional

Para hacer la comparación de género se tomaron en cuenta los resultados que se presentan en el artículo original de Marlow, Marlow y Arnold (1995), los cuales muestran la importancia que se le da al programa de oportunidades de desarrollo o plan de carrera; posteriormente, se realizó un análisis con los resultados obtenidos de la muestra mexicana (ver cuadro 1).

Cuadro 1

Comparativo de los diversos criterios considerados en el desarrollo profesional de acuerdo con el género de los participantes

\begin{tabular}{|c|c|c|c|c|c|c|}
\hline \multirow[t]{4}{*}{ Criterio } & \multicolumn{6}{|c|}{$\begin{array}{l}\text { Nivel de importancia } \\
\text { 1. No es importante y } 5 \text {. Sí es muy importante }\end{array}$} \\
\hline & \multicolumn{2}{|c|}{ Mujeres } & \multicolumn{4}{|c|}{ Hombres } \\
\hline & & & Dif. & & & \\
\hline & EUA & $\begin{array}{l}\text { MEX } \\
n=181\end{array}$ & $\begin{array}{l}\text { México } \\
v s .\end{array}$ & EUA & $\begin{array}{l}\text { MEX } \\
n=167\end{array}$ & $\begin{array}{l}\text { México } \\
v s .\end{array}$ \\
\hline & & & EUA & & & EUA \\
\hline 1. Tener un mentor & 4.21 & 3.14 & & 3.58 & 3.18 & \\
\hline 2. Atractivo físico & 3.57 & 2.56 & & 2.75 & 2.44 & \\
\hline 3. Nivel educativo & 4.42 & 4.48 & * & 3.87 & 4.40 & $*$ \\
\hline 4. Experiencia & 4.51 & 4.04 & & 3.80 & 3.79 & \\
\hline 5. Asesor de carrera & 2.99 & 3.23 & $*$ & 2.48 & 3.21 & $*$ \\
\hline 6. Apoyo ambiental del corporativo & 4.48 & 4.00 & & 3.29 & 3.63 & $*$ \\
\hline 7. Habilidades interpersonales & 4.58 & 4.28 & & 3.64 & 4.20 & $*$ \\
\hline 8. Habilidades de comunicación & 4.58 & 4.45 & & 3.96 & 4.31 & $*$ \\
\hline $\begin{array}{l}\text { 9. Disponibilidad de cambio de } \\
\text { residencia }\end{array}$ & 3.40 & 3.10 & & 3.38 & 3.24 & \\
\hline 10. Red de contactos laboral & 3.85 & 3.74 & & 3.65 & 3.65 & \\
\hline 11. Disponibilidad de viajar & 3.85 & 3.50 & & 3.72 & 3.56 & \\
\hline 12. Apoyo familiar & 4.48 & 4.40 & & 3.36 & 4.24 & $*$ \\
\hline $\begin{array}{l}\text { 13. Habilidad para adoptar valores de } \\
\text { tu supervisor }\end{array}$ & 3.65 & 3.74 & * & 3.47 & 3.62 & * \\
\hline 14. Capacidad de correr riesgos & 3.98 & 4.01 & $*$ & 3.76 & 3.89 & $*$ \\
\hline 15. Título universitario & 3.24 & 3.55 & $*$ & 2.85 & 3.62 & $*$ \\
\hline 16. Imagen profesional & 4.54 & 4.08 & & 3.82 & 3.99 & $*$ \\
\hline $\begin{array}{l}\text { 17. Habilidad de cambios de patrones } \\
\text { de vida }\end{array}$ & 3.74 & 3.59 & & 3.15 & 3.62 & * \\
\hline $\begin{array}{l}\text { 18. Habilidades para equilibrar activi- } \\
\text { dades profesionales y familiares }\end{array}$ & 4.62 & 4.39 & & 2.80 & 4.08 & $*$ \\
\hline
\end{tabular}

Fuente: Elaboración propia, con los datos de la muestra de EUA de Marlow, Marlow y Arnold (1995). 
Desde la perspectiva de las mujeres mexicanas, se observa que en cinco de los 18 criterios analizados para el desarrollo profesional es mayor el grado de importancia que ellas le otorgan con respecto a las estadounidenses, mientras que para los hombres en 12 criterios se presentan promedios mayores que en la muestra original. Sin duda, sabemos de la relevancia que tiene la preparación académica de las personas y la obtención de un título profesional para el éxito en su carrera, así como de las habilidades para relacionarse con el jefe inmediato en el ámbito laboral; es por esto que las empresas deben enfocarse en procesos de planeación de recursos humanos y en la asesoría del personal clave, pues esto repercutirá en el crecimiento de la organización.

En los resultados de los análisis de las varianzas (ANOVAS) realizados únicamente para la muestra mexicana podemos destacar que en sólo tres criterios se presentan diferencias estadísticamente significativas entre los hombres y las mujeres mexicanas: 1) experiencia, $\mathrm{F}=6.38$ con un nivel de significancia de 0.011978 ; 2) apoyo ambiental del corporativo que también puede ser analizado desde la perspectiva del apoyo organizacional, $\mathrm{F}=14.28$ con 0.000185 ; y 3 ) las habilidades para equilibrar las actividades profesionales y familiares, $\mathrm{F}=10.83$ con 0.001101 de nivel de significancia. Las mujeres le dan mayor importancia a uno de estos criterios.

Comparación de mujeres y hombres en EUA y México de acuerdo con el nivel de importancia de los factores de desarrollo profesional

Se realizó un análisis de comparación de acuerdo con el promedio de la importancia que les dan las mujeres a los diversos criterios del desarrollo profesional, donde "1" No es importante y "5" Sí es muy importante. Es necesario mencionar que dentro de los cinco primeros criterios considerados por las mujeres, existen tres aspectos en común aunque aparecen en diferente orden: 1) habilidades para equilibrar las actividades profesionales y laborales, 2) habilidades interpersonales y 3 ) habilidades de comunicación (ver cuadro 2). 
Cuadro 2

Clasificación de los criterios de desarrollo profesional desde la perspectiva femenina

\begin{tabular}{|c|c|c|c|c|}
\hline Lugar & & EUA & & México \\
\hline 1 & $\begin{array}{l}\text { 18. Habilidades para equilibrar } \\
\text { actividades profesionales } \\
\text { y familiares }\end{array}$ & 4.62 & 3. Nivel educativo & 4.48 \\
\hline 2 & 7. Habilidades interpersonales & 4.58 & 8. Habilidades de comunicación & 4.45 \\
\hline 3 & $\begin{array}{l}\text { 8. Habilidades } \\
\text { de comunicación }\end{array}$ & 4.58 & 12. Apoyo familiar & 4.40 \\
\hline 4 & 16. Imagen profesional & 4.54 & $\begin{array}{l}\text { 18. Habilidades para equilibrar } \\
\text { actividades profesionales } \\
\text { y familiares }\end{array}$ & 4.39 \\
\hline 5 & 4. Experiencia & 4.51 & 7. Habilidades interpersonales & 4.28 \\
\hline
\end{tabular}

Fuente: Elaboración propia con los datos de la muestra de EUA de Marlow, Marlow y Arnold (1995).

Estos factores permiten observar la relevancia de una serie de habilidades que deben desarrollarse en las personas y que deben fortalecerse por medio un programa de capacitación con competencias profesionales o con planes de carrera.

El cuadro 3 presenta el promedio de la muestra donde " 1 " No es importante y "5" Sí es muy importante. Se puede observar la clasificación que le dan los hombres en ambas muestras; es importante destacar que para los mexicanos los criterios encontrados son los mismos consideran las mujeres, pero únicamente con diferente orden. Para el caso de la muestra norteamericana, sólo tres criterios fueron similares. 
Cuadro 3

Clasificación de los criterios de desarrollo profesional desde la perspectiva masculina

\begin{tabular}{|c|c|c|c|c|}
\hline Lugar & Hombres & EUA & & México \\
\hline 1 & 8. Habilidades de comunicación & 3.96 & 3. Nivel educativo & 4.40 \\
\hline 2 & 3. Nivel educativo & 3.87 & 8. Habilidades de comunicación & 4.31 \\
\hline 3 & 16. Imagen profesional & 3.82 & 12. Apoyo familiar & 4.24 \\
\hline 4 & 4. Experiencia & 3.80 & 7. Habilidades interpersonales & 4.20 \\
\hline 5 & 14. Capacidad de correr riesgos & 3.76 & $\begin{array}{l}\text { 18. Habilidades para equilibrar } \\
\text { actividades profesionales } \\
\text { y familiares }\end{array}$ & 4.08 \\
\hline
\end{tabular}

Fuente: Elaboración propia con los datos de la muestra de EUA de Marlow, Marlow y Arnold (1995).

En este apartado el apoyo familiar es importante para la muestra mexicana pues aparece dentro de los factores más relevantes, mientras que para los estadounidenses no; con ello nos damos cuenta del valor que tiene la familia en el desarrollo profesional y en la cultura mexicana.

Comparación de mujeres y hombres de acuerdo con los criterios de compensaciones monetarias

A continuación se presentan los resultados de los diversos criterios en las compensaciones de acuerdo con la importancia que le dan las mujeres y los hombres (ver cuadros 4 y 5). Como se observa, tanto en las compensaciones monetarias como en las no monetarias existen diferencias estadísticamente significativas entre las mujeres y los hombres. Lo más destacable es que todas las compensaciones son más importantes para las mujeres que para los hombres. 


\section{Cuadro 4}

Análisis comparativo por género de los criterios de las compensaciones monetarias

\begin{tabular}{lcccc}
\hline \multicolumn{1}{c}{ Criterio } & \multicolumn{4}{c}{ Nivel de importancia } \\
1. No es importante y 5. Si es muy importante \\
\hline Compensaciones monetarias & $\begin{array}{c}\text { Mujeres } \\
\text { MEX 181 }\end{array}$ & $\begin{array}{c}\text { Hombres } \\
\text { MEX 167 }\end{array}$ & ANOVA & Signif. \\
19. Sueldo base & 4.27 & 3.86 & $* \mathrm{~F}=19.13$ & 0.000016 \\
20. Prestaciones & 4.39 & 4.0 & $* \mathrm{~F}=19.02$ & 0.000017 \\
21. Bonos & 4.15 & 3.89 & $* \mathrm{~F}=6.98$ & 0.008630 \\
\hline
\end{tabular}

De los tres componentes que tienen las compensaciones monetarias, las prestaciones son las que presentan mayor importancia en ambos casos; por esta razón, las empresas deben prestar especial atención a este rubro y no hacer caso omiso, ni reducir sus presupuestos para la reducción de prestaciones, pues son un factor muy importante para los trabajadores.

El cuadro 5 muestra que los aspectos más importantes para las mujeres son la capacitación, el ambiente laboral y las oportunidades de carrera en las empresas. Desde nuestra perspectiva, se pueden asociar como una compensación monetaria relevante en la actualidad, que formar parte de una estrategia empresarial en los procesos de atracción y retención del talento humano. 


\section{Cuadro 5}

Análisis comparativo por género de los criterios de las compensaciones no monetarias

\begin{tabular}{ccccc}
\hline Criterio & \multicolumn{4}{c}{ Nivel de importancia } \\
1. No es importante y 5. Si es muy importante \\
\hline $\begin{array}{c}\text { Compensaciones } \\
\text { no monetarias } \\
\text { 22. Capacitación }\end{array}$ & $\begin{array}{c}\text { Mujeres } \\
\text { MEX 181 }\end{array}$ & $\begin{array}{c}\text { Hombres } \\
\text { MEX 167 }\end{array}$ & ANOVA & Signif. \\
23. Reconocimiento & 4.61 & 4.37 & $* \mathrm{~F}=10.89$ & 0.001065 \\
24. Autonomía & 4.34 & 4.16 & $* \mathrm{~F}=4.15$ & 0.042519 \\
25. Calidad de vida & 4.33 & 4.04 & $* \mathrm{~F}=11.62$ & 0.000729 \\
& 4.45 & 4.23 & $* \mathrm{~F}=7.07$ & 0.008192 \\
26. Ambiente laboral & 4.48 & 4.26 & $* \mathrm{~F}=8.46$ & 0.003867 \\
& 4.48 & 4.24 & $* \mathrm{~F}=8.46$ & 0.003867 \\
\hline
\end{tabular}

Factores relevantes de acuerdo con el nivel de importancia que tienen para la muestra mexicana

En este apartado se muestran los factores de cada uno de los componentes del modelo que tienen mayor promedio de acuerdo con el nivel de importancia que le dan las personas, donde "1" No es importante y "5" Sí es muy importante.

Por lo que respecta al grupo de los factores de desarrollo profesional, de acuerdo con el promedio obtenido, los criterios más importantes son: nivel educativo (4.44), habilidades de comunicación (4.38), apoyo familiar (4.32), habilidades de saber equilibrar la demanda laboral del trabajo con las actividades familiares (4.24) y habilidades interpersonales (4.24). Por otra parte, los cinco aspectos de menor importancia fueron: el atractivo físico, tener un mentor, disponibilidad de cambio de residencia, asesor de carrera y disponibilidad para viajar.

Con respecto al grupo de los factores de las compensaciones monetarias, de acuerdo con el promedio obtenido, las personas dan importancia a las prestaciones (4.20), el sueldo base (4.07) y los bonos (4.02); en cambio, los factores de las com- 
pensaciones no monetarias más relevantes son la capacitación (4.49), el ambiente laboral (4.37), las oportunidades de carrera (4.36) y la calidad de vida (4.35).

\section{Agrupación de variables}

Para el análisis de este aspecto, se agruparon diversas variables del modelo de la investigación con la finalidad de obtener una serie de constructos relevantes que servirán de apoyo y para poder cumplir los objetivos previamente definidos. Se obtuvieron siete variables: 1) Competencias profesionales, 2) Imagen personal, 3) Disposición al cambio, 4) Asesoría, 5) Apoyo organizacional, 6) Apoyo familiar y 7) Red de contactos. En la figura 2 se observan las agrupaciones realizadas de los 18 factores relacionados con el desarrollo profesional; asimismo, se muestran los coeficientes de alfa cronbach que resultaron del análisis estadístico correspondiente.

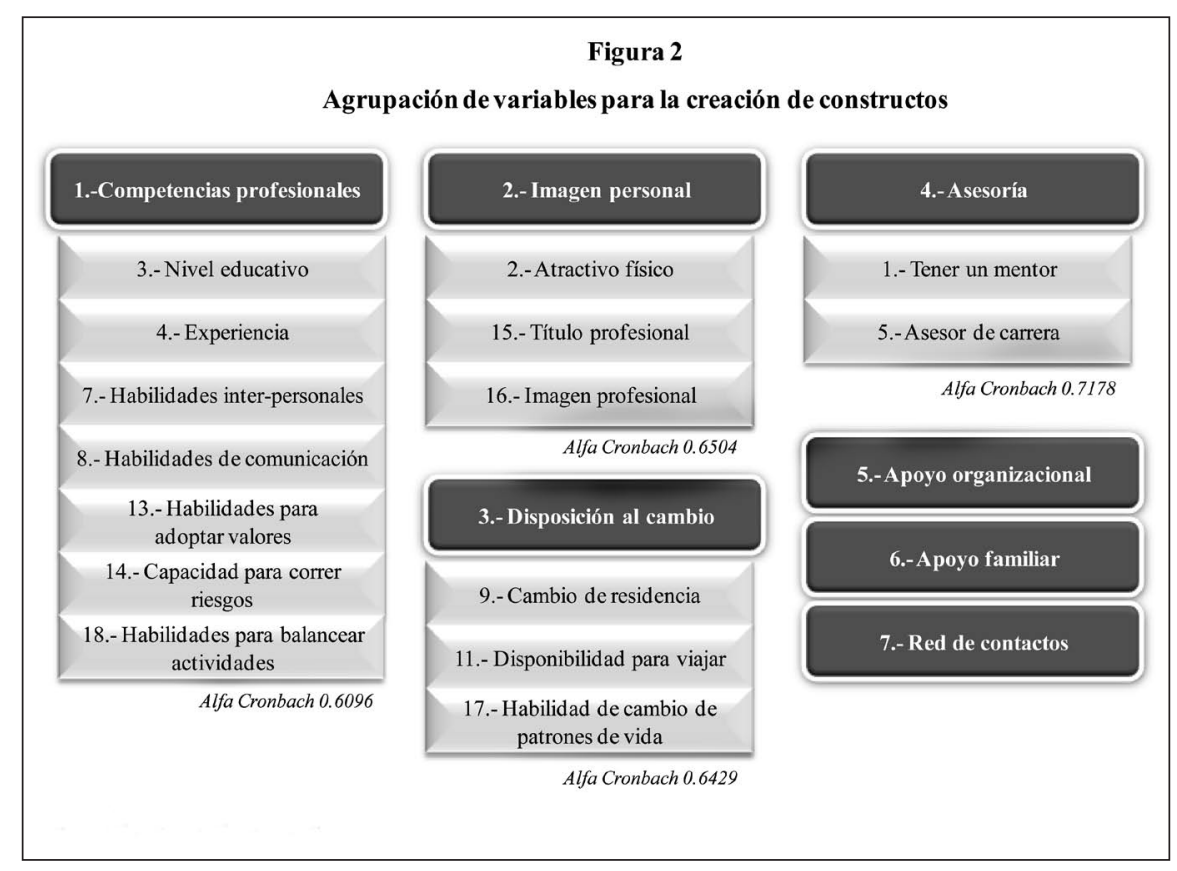

\section{Correlación entre las variables}

De este análisis de correlación entre las variables, las que presentan mayor relación con las compensaciones monetarias son el apoyo organizacional $(0.345845)$ y las 
competencias profesionales (0.347335). Además, esas mismas variables presentan mayores coeficientes de correlación para las compensaciones no monetarias; es decir, para el apoyo organizacional $(0.434561)$ y las competencias profesionales (0.489018). En todos los casos con niveles de significancia < al 0.005. La matriz de correlación resultante se presenta en el cuadro 6 en la sección de anexos.

\section{Conclusiones}

El nivel de importancia de los diversos criterios analizados con respecto al desarrollo profesional es diferente para los estadounidenses y los mexicanos, lo que muestra una diversidad cultural y profesional. En primer lugar, los valores en el desarrollo profesional que las mujeres de Estados Unidos presentaron fue diferente al de las mexicanas; esto es, el valor más relevante coincide en primer lugar con la habilidad de saber equilibrar la demanda laboral y del hogar; en segundo lugar, para las mujeres mexicanas es más importante el apoyo familiar, en cambio para las de Estados Unidos son las habilidades de comunicación; las mujeres de Estados Unidos le asignaron sexto lugar al apoyo familiar. Esto indica el significado que tiene la familia para los mexicanos

Asimismo, se puede observar que sólo el valor de "apoyo familiar", que se encuentra en el segundo lugar de la tabla en orden de importancia, coincide entre las mujeres y hombres de México.

Por otra parte, el nivel de importancia en la tabla de valores del desarrollo profesional no es igual para las mujeres y los hombres en Estados Unidos y en México; en los resultados se observa que el valor de "habilidad de saber equilibrar la demanda laboral y del hogar" se encuentra en primer lugar para las mujeres de ambos países; mientras que para el hombre de Estados Unidos lo más importante son las "habilidades de comunicación" y para el hombre de México es el "nivel educativo".

En cuanto a las recompensas monetarias, éstas son más importantes que los factores del desarrollo y que las recompensas no monetarias. Para los profesionistas mexicanos son más importantes las recompensas no monetarias que las monetarias; sin embargo, son más importantes los factores de desarrollo profesional.

En los resultados generales se percibe que las oportunidades de desarrollo de carrera son diferentes en ambos géneros; pero en lo relacionado con las compensaciones, éstas son más importantes para las mujeres que para los hombres. 
Cabe aclarar que los programas de desarrollo que se diseñen y se desarrollen para las mujeres en las organizaciones no deben ser copias de otros programas similares, sino que deben estar diseñados especialmente para las necesidades de las mujeres que se encuentran trabajando; además, se debe ubicar la etapa en que se tiene el desarrollo profesional; para ello, se debe destacar la experiencia para desempeñar sus funciones, las habilidades para equilibrar las actividades profesionales y familiares, así como el apoyo que se tenga de la empresa misma.

Por otra parte, los encuestados prefieren un ambiente agradable de trabajo, que es considerado como un componente de las compensaciones no monetarias, antes que los aspectos monetarios y el desarrollo profesional.

Entre las limitantes que se encontraron en el desarrollo de esta investigación y que son importantes considerar para trabajos próximos están: 1) modificar el instrumento de medición con el fin de profundizar en el conocimiento de las causas de esos criterios; 2) realizar análisis estadísticos para considerar las variables demográficas como estado civil y edad; 3) aplicar el instrumento de medición en diferentes regiones geográficas de México para poder realizar comparaciones por zonas y regiones; 4) relacionar los diversos criterios con las variables de satisfacción, motivación, calidad de vida, etcétera; y 5) considerar los 27 factores o algunos otros para identificar los predictores del éxito profesional, utilizando diversas pruebas estadísticas.

\section{Referencias}

Andrew, C., Coderre, C. and Denis, A. (1990). "Stop or Go: Reflections of Women Managers on Factors Influencing Their Career Development". Journal of Business Ethics. 9(4-5), 361-367.

Bohlander, G. W., Snell, S. A. and Sherman, Jr. A.W. (2001) Administración de Recursos Humanos. 12a. ed., México: Thompson Learning.

Brennan, M. (1992). "Marriage, Gender Influence Career Advancement for Chemists". Chemical \& Engineering News, 70(4), 46-51. 
Caligiuri, P. and Cascio, W. F. (2000). "Sending women on global assignments: Challenges, myths and solutions". WorldatWork Journal. 9(2), 34-41

Cascio, F. (2003). Managing Human Resources-productivity, Quality of Work life profits. $6^{\text {th }}$ edition, New York: McGraw-Hill Higher Education.

Chiavenato, I. (2007). Administración de los recursos humanos. 8a. ed., México: Mc Graw Hill.

Dalton, G. W., Thompson, P. H. and Price, R. L. (1977). "The Four Stages of Professional Careers-A New Look at Performance by Professionals". Organizational Dynamics. 6(1), 19-42.

Gómez-Mejía, L.R., Balkin, D. B. and Cardy, R.L. (2001). Dirección y gestión de Recursos Humanos. $3^{\text {a }}$. ed., Madrid: Pearson Educación.

Greenhaus, J. H., Parasuraman, S. and Wormley, W. M. (1990). "Effects of Race on Organizational Experiences, Job Performance Evaluations, and Career". Academy of Management Journal. 33(1), 64-86.

Hernández-Sampieri, R., Fernández, C. and Baptista, P. (2006). Metodología de la investigación. 4a. ed., México: McGraw-Hill Interamericana.

Igbaria, M. and Greenhaus, J. H. (1992). "The Career Advancement Prospects of Managers and Professionals: Are MIS Employees Unique?" Decision Sciences. 23(2),478-499

Igbaria, M. and Shayo, C. (1997). "The impact of race and gender differences on job performance evaluations and career success". Equal Opportunities International. 16(8), 12-23.

Jaffe, B. (1985). "A Forced Fit (Career Development of Women Executives)". Training and Development Journal. 39, 82-83.

Kaye, B. and Jacobson, B. (1996). "Reframing Mentoring". Training \& Development . 50(8), 44-47. 
Kelly, R. M. and Guy, M. E. (1991). "Public Managers in the States: A Comparison of Career Advancement by Sex". Public Administration Review. 51(5), 402-412.

Madero, S. M. (2009). "Factores de atracción y retención del mercado laboral, para empleados potenciales". Economía, Gestión y Desarrollo. 7, 131147.

Margol, I. (1993). “CoManaging Career Paths". Training \& Development, 47, 1113.

Marlow, N. D., Marlow, E. K. and Arnold, V. A. (1995). "Career development and women managers: Does one size fit all"? HR Human Resource Planning. 18(2), 38-49.

McCloskey, D. W. and Igbaria, M. (2003). Does 'out of sight' mean 'out of mind'? An empirical investigation of the career advancement prospects of telecommuters". Information Resources Management Journal. 16(2), 19-34.

Menefee, J. A. and Murphy, R.O. (2004) "Rewarding and retaining the best: Compensation strategies for top performers". Benefits Quarterly. 20(3), 13-20.

Mondy, R.W. and Noé, R. M. (2005) Administración de Recursos Humanos. $9^{\mathrm{a}}$. ed., México: Pearson Prentice Hall.

Mowday, R. T., Porter, L.W. and Steers, R. M. (1982). Employee-organization linkages: The psychology of commitment, absenteeism, and turnover. New York: Academic Press.

Naff, K.C. (1993). "Through the glass ceiling: Prospects for the advancement of women in the federal civil service". Public Administration Review. 54(6), 507-514.

Roche, G. R (1979). "Much Ado about Mentors". Harvard Business Review. 57. 14-28.

Schuster, J. R. and Zingheim, P.K. (1993). "The new variable pay: Key design issues". Compensation and Benefits review. 25(2), 27-34. 
Silverman, L. (2006). "How Do You Keep the Right People on the Bus? Try Stories". The Journal for Quality and Participation. 29(4), 11-15.

Syed T. H., Adeel A. and Syed A. M. (2007) "Impact of Non-Financial Rewards on Employee Motivation: (A case of Cellular Communication Service providing sector of Telecom Industry registered under PTA in Islamabad)". The Business Review, Cambridge. 7(2), 272-277.

Wentling, R. M. (1992). "Women in Middle Management: Their Career Development and Aspirations". Business Horizons. 35, 47-54.

(1993). "Women Managers: Room at the Top?" Industry Week, 242(5), 58-61.

Zobal, C. (1998). "The 'ideal' team compensation system-an overview, part I". Team Performance Management. 4(5), 235-249.

(1999) "The 'ideal' team compensation system-an overview, part II". Team Performance Management. 5(1), 23-45. 
Anexos

Cuadro 1

Características demográficas de la muestra

\begin{tabular}{|c|c|c|c|c|c|c|c|c|c|c|c|c|c|c|}
\hline \multirow[b]{3}{*}{ Variable } & & \multicolumn{3}{|c|}{ Edad } & \multirow{2}{*}{\multicolumn{3}{|c|}{$\frac{\text { Experiencia }}{\text { laboral }}$}} & \multirow{2}{*}{\multicolumn{3}{|c|}{$\frac{\text { Experiencia }}{\text { en el puesto }}$}} & \multicolumn{4}{|c|}{ Nivel jerárquico } \\
\hline & & \multirow[b]{2}{*}{ 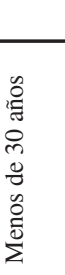 } & \multirow[b]{2}{*}{ 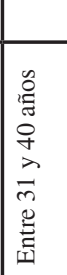 } & \multirow[b]{2}{*}{ 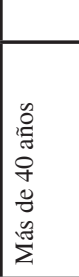 } & & & & & & & & & & \\
\hline & & & & & 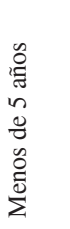 & 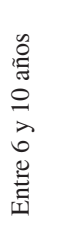 & 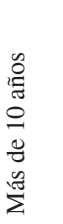 & 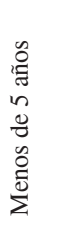 & 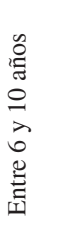 & 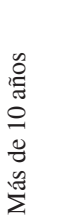 & 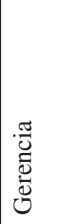 & 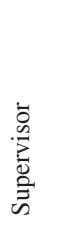 & 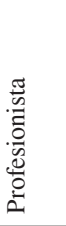 & 导 \\
\hline \multirow{2}{*}{$\begin{array}{l}\text { Mascu- } \\
\text { lino }\end{array}$} & 167 & 38 & 81 & 48 & 87 & 42 & 38 & 102 & 46 & 19 & 56 & 26 & 35 & 50 \\
\hline & $48 \%$ & $23 \%$ & $49 \%$ & $29 \%$ & $52 \%$ & $25 \%$ & $23 \%$ & $61 \%$ & $28 \%$ & $11 \%$ & $34 \%$ & $16 \%$ & $21 \%$ & $30 \%$ \\
\hline \multirow{2}{*}{$\begin{array}{l}\text { Femeni- } \\
\text { no }\end{array}$} & 181 & 68 & 86 & 27 & 111 & 38 & 32 & 134 & 36 & 11 & 22 & 40 & 70 & 49 \\
\hline & $52 \%$ & $38 \%$ & $48 \%$ & $15 \%$ & $61 \%$ & $21 \%$ & $18 \%$ & $74 \%$ & $20 \%$ & $6 \%$ & $12 \%$ & $22 \%$ & $39 \%$ & $27 \%$ \\
\hline \multirow[t]{5}{*}{ Total } & 348 & 106 & 167 & 75 & 198 & 80 & 70 & 236 & 82 & 30 & 78 & 66 & 105 & 99 \\
\hline & & $30 \%$ & $48 \%$ & $22 \%$ & $57 \%$ & $23 \%$ & $20 \%$ & $68 \%$ & $24 \%$ & $9 \%$ & $22 \%$ & $19 \%$ & $30 \%$ & $28 \%$ \\
\hline & & \multicolumn{3}{|c|}{ Chi-Square 13.9796} & \multicolumn{3}{|c|}{ Chi-Square 3.0651} & \multicolumn{3}{|c|}{ Chi-Square 7.1401} & \multicolumn{4}{|c|}{ Chi-Square 28.9506} \\
\hline & & \multicolumn{3}{|c|}{ GL 2} & \multicolumn{3}{|c|}{ GL 2} & \multicolumn{3}{|c|}{ GL 2} & \multicolumn{4}{|c|}{ GL 3} \\
\hline & & \multicolumn{3}{|c|}{$\begin{array}{c}\text { Prob. Level } \\
0.000921\end{array}$} & \multicolumn{3}{|c|}{$\begin{array}{c}\text { Prob. Level } \\
0.215982\end{array}$} & \multicolumn{3}{|c|}{$\begin{array}{c}\text { Prob. Level } \\
0.028154\end{array}$} & \multicolumn{4}{|c|}{ Prob. Level 0.000002} \\
\hline
\end{tabular}




\section{Cuadro 6}

\section{Matriz de correlación}

\section{Pearson Correlations Section (Row-Wise Deletion)}

\begin{tabular}{llrcccc}
\hline & $\begin{array}{l}\text { apoyo_org } \\
\text { apoyo_org }\end{array}$ & red_contactos & apoyo_familiar & compet_prof & imágen & disposición \\
red_contactos & 0.217268 & 1.000000 & & & & \\
$\begin{array}{l}\text { apoyo_familiar } \\
\text { compet_prof }\end{array}$ & 0.280362 & 0.219133 & 1.000000 & & & \\
& 0.389006 & 0.379447 & 0.389892 & 1.000000 & & \\
imágen & 0.162436 & 0.297907 & 0.209782 & 0.407241 & 1.000000 & \\
disposición & 0.159061 & 0.486340 & 0.257606 & 0.502477 & 0.426564 & 1.000000 \\
asesoría & 0.334191 & 0.271338 & & 0.246637 & 0.151553 & 0.232815 \\
monetarias & 0.345845 & 0.233633 & 0.226256 & 0.347335 & 0.233837 & 0.167922 \\
nomonetarias & 0.434561 & 0.252993 & 0.293898 & 0.489018 & 0.226674 & 0.193979
\end{tabular}

Cronbachs Alpha $=0.766553$ Standardized Cronbachs Alpha $=0.786219$

\section{Pearson Correlations Section (Row-Wise Deletion)}

\begin{tabular}{lccc}
\hline & asesoría & monetarias & nomonetarias \\
$\begin{array}{l}\text { apoyo_org } \\
\text { red_contactos } \\
\text { apoyo_familiar } \\
\text { compet_prof } \\
\text { imágen }\end{array}$ & & \\
disposición & 1.000000 & & \\
asesoría & 1.000000 & 0.586308 & \\
monetarias & 0.197002 & 0.586308 & 1.000000 \\
nomonetarias & & \\
Cronbachs Alpha $=0.766553$ & Standardized Cronbachs Alpha $=0.786219$
\end{tabular}

